Pacific

Journal of

Mathematics

\title{
MULTIPLICITÉ DU SPECTRE DE STEKLOV SUR LES SURFACES ET NOMBRE CHROMATIQUE
}

PierRe JAMMES

Volume $282 \quad$ No. 1

May 2016 


\title{
MULTIPLICITÉ DU SPECTRE DE STEKLOV SUR LES SURFACES ET NOMBRE CHROMATIQUE
}

\author{
PIERRE JAMMES
}

\begin{abstract}
On démontre plusieurs résultats sur la multiplicité des premières valeurs propres de Steklov sur les surfaces compactes à bord. On améliore certaines bornes sur la multiplicité, en particulier pour la première valeur propre, et on montre qu'elles sont optimales sur plusieurs surfaces de petit genre. Dans un article précédent, on a défini un nouvel invariant chromatique des surfaces à bord et on a conjecturé qu'il est relié à la multiplicité de la première valeur propre de Steklov. Dans le present article on étudie cet invariant et on démontre une des inégalités de la conjecture.

We prove several results about the multiplicity of the first Steklov eigenvalues on compact surfaces with boundary. We improve some bounds on the multiplicity, especially for the first eigenvalue, and we prove they are sharp on some surfaces of small genus. In a previous article, we defined a new chromatic invariant of surfaces with boundary and conjectured that this invariant is related to the bound on the first eigenvalue. In the present article, we study this invariant, and prove one of the inequalities of this conjecture.
\end{abstract}

\section{Introduction}

L'étude de la multiplicité des valeurs propres de Steklov a fait récemment l'objet des travaux [Fraser et Schoen 2015; Jammes 2014; Karpukhin et al. 2014]. Ces trois articles montrent que sur une surface compacte à bord donnée, la multiplicité de la $k$-ième valeur propre de Steklov est majorée en fonction de $k$ et de la topologie (voir le paragraphe qui suit pour la définition du spectre de Steklov). Dans [Jammes 2014], j'ai montré que ce phénomène est spécifique à la dimension 2 et qu'en dimension plus grande, on peut prescrire arbitrairement le début du spectre de Steklov, avec multiplicité. J'ai aussi conjecturé qu'en dimension 2, la multiplicité maximale de la première valeur propre non nulle est déterminée par un invariant topologique de même nature que le nombre chromatique mais spécifique aux surfaces à bord (cf. la conjecture 1.6 ci dessous).

MSC2010: 35P15, 57M15, 58J50.

Mots-clefs: Steklov eigenvalues, multiplicity, chromatic number. 
L'objet de cet article est triple. D'abord, améliorer certaines bornes sur la multiplicité du spectre de Steklov en dimension 2, en particulier pour la première valeur propre et sur les surfaces de petit genre. Ensuite, construire des exemples de première valeur propre multiple, ce qui permet de montrer que certaines bornes sont optimales. Enfin, on étudie l'invariant chromatique des surfaces à bord définie dans [Jammes 2014], qu'on appellera nombre chromatique relatif, on justifiera en particulier cette dénomination et on calculera sa valeur sur les surfaces de petit genre.

La confrontation de ces différents résultats permet de consolider la conjecture faite dans [Jammes 2014] sur le lien entre la multiplicité de la première valeur propre et le nombre chromatique relatif en montrant qu'elle est vérifiée dans tous les cas où on connaît la borne optimale et qui sont résumés dans la table 1 ci-dessous.

1A. Définitions et notations. Avant d'énoncer les résultats précis, nous allons rappeler les notions en jeu et préciser quelques définitions.

Soit $M$ une variété compacte à bord et $\gamma \in C^{\infty}(M), \rho \in C^{\infty}(\partial M)$ deux fonctions densités strictement positives sur $M$ et $\partial M$ respectivement (on peut travailler avec des hypothèses de régularité plus faible sur les densités mais ça n'est pas crucial dans la suite). Le problème aux valeurs propres de Steklov consiste à résoudre l'équation, d'inconnues $\sigma \in \mathbb{R}$ et $f: \bar{M} \rightarrow \mathbb{R}$,

$$
\begin{cases}\operatorname{div}(\gamma \nabla f)=0 & \text { dans } M, \\ \gamma \frac{\partial f}{\partial v}=\sigma \rho f & \text { sur } \partial M,\end{cases}
$$

où $v$ est un vecteur unitaire sortant normal au bord. On parle de problème de Steklov homogène quand $\gamma \equiv 1$ et $\rho \equiv 1$, et le cas $\gamma \neq \equiv 1$ se rattache au problème de Calderón. L'ensemble des réels $\sigma$ solutions du problème forme un spectre discret positif noté

$$
0=\sigma_{0}(M, g, \rho, \gamma)<\sigma_{1}(M, g, \rho, \gamma) \leq \sigma_{2}(M, g, \rho, \gamma) \leq \cdots
$$

On omettra les références à $\rho$ et $\gamma$ quand ces densités sont uniformément égales à 1 .

On s'intéressera à la multiplicité de ces valeurs propres, et si on se donne une surface compacte à bord $\Sigma$, on notera $m_{k}(\Sigma)$ la multiplicité maximale de $\sigma_{k}(\Sigma, g, \rho, \gamma)$ quand on fait varier $g, \rho$ et $\gamma$.

On établira des liens entre ce spectre et les graphes plongés dans la surface. On introduit pour cela les deux définitions suivantes :

Définition 1.3. Soit $\Gamma$ un graphe fini et $\Sigma$ une surface compacte à bord. Un plongement de $\Gamma$ dans $\Sigma$ sera appelé plongement propre si ce plongement envoie tous les sommets de $\Gamma$ sur $\partial \Sigma$.

Remarque 1.4. Les graphes qui se plongent proprement dans le disque sont connus sous le nom de graphes planaires extérieurs (outerplanar graphs). 
Définition 1.5. Si $\Sigma$ est une surface compacte à bord, on appelle nombre chromatique relatif de $\Sigma$, noté $\operatorname{Chr}_{0}(\Sigma)$, la borne supérieure des nombres chromatiques des graphes finis qui admettent un plongement propre dans $\Sigma$.

Avec ces notations, la conjecture énoncée dans [Jammes 2014] peut se reformuler ainsi (l'équivalence entre les deux énoncés est l'un des objets de la section 3, cf. remarque 3.4) :

Conjecture 1.6. Pour toute surface $\Sigma$ compacte à bord, on a

$$
m_{1}(\Sigma)=\operatorname{Chr}_{0}(\Sigma)-1 \text {. }
$$

Cette conjecture adapte au spectre de Steklov une conjecture analogue énoncée par Y. Colin de Verdière dans [Colin de Verdière 1987] et reliant la multiplicité de la 2-ième valeur propre des opérateurs de Schrödinger et le nombre chromatique usuel.

Enfin, on utilisera les notations suivantes : $\mathbb{S}^{2}, \mathbb{D}^{2}, \mathbb{P}^{2}, \mathbb{M}^{2}, \mathbb{T}^{2}$ et $\mathbb{K}^{2}$ désigneront respectivement la sphère de dimension 2 , le disque, le plan projectif, le ruban de Möbius, le tore et la bouteille de Klein. Si $\Sigma$ est une surface close, on désignera par $\Sigma_{p}$ la surface $\Sigma$ privée de $p$ disques disjoints, où $p$ est un entier strictement positif. Par exemple, on a $\mathbb{M}^{2}=\mathbb{P}_{1}^{2}$ et $\mathbb{D}^{2}=\mathbb{S}_{1}^{2}$.

1B. Résultats. Un premier résultat de cet article est de démontrer qu'on a au moins une inégalité dans l'égalité de la conjecture 1.6 :

Théorème 1.7. Pour toute surface $\Sigma$ une surface compacte à bord, on a

$$
m_{1}(\Sigma) \geq \operatorname{Chr}_{0}(\Sigma)-1 \text {. }
$$

On va s'attacher ensuite à améliorer les bornes connues sur la multiplicité des valeurs propres, en commençant par considérer une surface quelconque. Rappelons d'abord le résultat de [Karpukhin et al. 2014], qui est la meilleure majoration connue pour une surface $\Sigma$ et une valeur propre $\sigma_{k}$ quelconques (elle est démontré dans [Karpukhin et al. 2014] pour $\gamma \equiv 1$ mais sans hypothèse de régularité sur $\rho$ ) :

Théorème 1.8 [Karpukhin et al. 2014]. Si $\Sigma$ est une surface compacte à bord de caractéristique d'Euler $\chi$ et dont le bord possède l composantes connexes, alors

$$
m_{k}(\Sigma) \leq 2 k-2 \chi-2 l+5
$$

et

$$
m_{k}(\Sigma) \leq k-2 \chi+4
$$

pour tout $k \geq 1$, cette dernière égalité étant stricte pour les surfaces non simplement connexes. 
Les majorations de multiplicité que nous allons montrer sont de plusieurs types. Dans les deux théorèmes qui suivent, nous donnons deux majorations de $m_{k}$ sur n'importe quelle surface : le premier précise l'inégalité (1.10) en utilisant les outils développés dans [Fraser et Schoen 2015], le deuxième théorème est une application des techniques développées par B. Sévennec [1994; 2002] pour majorer la multiplicité de la première valeur propre du laplacien. Nous donnerons ensuite des majorations spécifiques aux premières valeurs propres des surfaces de petit genre.

Théorème 1.11. Si $\Sigma$ est une surface compacte à bord de caractéristique d'Euler $\chi$ et dont le bord possède l composantes connexes, alors pour tout $k \geq 1$ :

$$
m_{k}(\Sigma) \leq k-2 \chi+3 .
$$

Remarque 1.13. La majoration (1.12) est optimale pour $k=1$ sur le disque et le ruban de Möbius, et dans les deux cas l'égalité est atteinte par un problème homogène, c'est-à-dire avec $\rho \equiv 1$ et $\gamma \equiv 1$ (pour la métrique canonique dans le cas du disque et pour une métrique $S^{1}$-invariante construite dans [Fraser et Schoen 2015] pour le ruban de Möbius).

Remarque 1.14. L'inégalité (1.12) étend les cas d'inégalité stricte de (1.10) à toutes les surfaces. Les techniques de [Fraser et Schoen 2015] qui autorisent cette démonstration unifiée permettent aussi les raffinements obtenus dans les théorèmes 1.20 et 1.21 .

Contrairement au théorème 1.11 et aux travaux [Fraser et Schoen 2015; Karpukhin et al. 2014; Jammes 2014], le théorème qui suit n'utilise pas les résultats de Cheng [1976] sur la structure de l'ensemble nodal des fonctions propres mais les techniques topologiques de Sévennec, ce qui autorise des hypothèses de régularité beaucoup plus faibles (voir le théorème 5.11 et la remarque 5.13).

Théorème 1.15. Soit $\Sigma$ une surface compacte à bord de caractéristique d'Euler $\chi$ et dont le bord possède l composantes connexes. Si $\chi+l \leq-1$, alors

$$
m_{1}(\Sigma) \leq 5-\chi-l \text {. }
$$

Si $l=1$ et $\chi \leq-2$, alors

$$
m_{1}(\Sigma) \leq 3-\chi
$$

Ces majorations restent valables si $g, \rho$ et $\gamma$ sont $L^{\infty}$ sans régularité supplémentaire.

Remarque 1.18. Si on note $\bar{\chi}$ la caractéristique d'Euler de la surface close obtenue en collant un disque sur chaque bord de $\Sigma$, la majoration (1.16) s'écrit $m_{1}(\Sigma) \leq 5-\bar{\chi}$. Elle est optimale pour $\bar{\chi}=-1$ et $l \geq 2$, et $\bar{\chi}=-2$ ou -3 et $l \geq 3$. L'inégalité (1.17) 


\begin{tabular}{|c|c|c|c|c|c|c|c|c|c|}
\hline$p=$ & 1 & 2 & 3 & 4 & $p=$ & 1 & 2 & 3 & 4 \\
\hline $\mathbb{S}^{2}$ & 2 & 3 & 3 & 3 & $\# 3 \mathbb{P}^{2}$ & 5 & 6 & 6 & 6 \\
\hline $\mathbb{P}^{2}$ & 4 & 4 & 5 & 5 & $\mathbb{T}^{2} \# \mathbb{\square}^{2}$ & 6 & 7 & 7 & 7 \\
\hline $\mathbb{K}^{2}$ & $?$ & 5 & 5 & 5 & $\# 4 \mathbb{P}^{2}$ & 6 & $?$ & 7 & 7 \\
\hline $\mathbb{\mathbb { T }}^{2}$ & 5 & $?$ & 6 & 6 & $\# 5 \mathbb{P}^{2}$ & 7 & $?$ & 8 & 8 \\
\hline
\end{tabular}

Table 1. Valeur de $m_{1}$ sur les surfaces de petit genre.

peut s'écrire $m_{1}(\Sigma) \leq 4-\bar{\chi}$, elle est optimale pour $\bar{\chi}=1,2$, 3. Les valeurs propres multiples correspondantes sont données par le théorème 1.7 et le calcul des nombres chromatiques relatifs fait dans la section 3 .

Dans les trois théorèmes qui suivent, on donne des bornes spécifiques aux surfaces de petit genre. Le premier adapte des résultats analogues obtenus par G. Besson [1980] et Colin de Verdière [1987] pour le laplacien.

Théorème 1.19. $m_{1}\left(\mathbb{T}_{p}^{2}\right)=6$ pour $p \geq 3$ et $m_{1}\left(\mathbb{K}_{p}^{2}\right)=5$ pour $p \geq 2$.

Avec des techniques similaires mais en utilisant des arguments spécifiques au spectre de Steklov, on améliore deux bornes :

Théorème 1.20. $m_{1}\left(\mathbb{P}_{2}^{2}\right)=4$ et $m_{1}\left(\mathbb{\nabla}_{1}^{2}\right)=5$.

Enfin, on majore la multiplicité de la 2-ième valeur propre du disque au moyen d'une stratégie indépendante de toutes celles évoquées jusqu'ici.

Théorème 1.21. $m_{2}\left(\mathbb{D}^{2}\right)=2$.

Remarque 1.22. On sait déjà que $m_{1}\left(\mathbb{D}^{2}\right)=2$. Par ailleurs, M. Karpukhin, G. Kokarev et I. Polterovich [2014] montrent que $m_{k}\left(\mathbb{D}^{2}\right)=2$ si $k$ est assez grand. Ces résultats laissent penser qu'on a $m_{k}\left(\mathbb{D}^{2}\right)=2$ quel que soit $k$. Cette multiplicité est atteinte par la métrique canonique.

La table 1 rassemble les valeurs connues de $m_{1}\left(\Sigma_{p}\right)$, où $\Sigma$ est une surface close (dans cette table, la notation $\# n \Sigma$ désigne la somme connexe de $n$ copies de la surface $\Sigma$ ). Pour les surfaces $\Sigma$ concernées, la valeur de $m_{1}$ ne dépend pas de $p$ quand $\mathrm{p} \geq 4$. Rappelons que la majoration de $\mathbb{S}_{p}^{2}$ pour $p \geq 2$ est montrée dans [Fraser et Schoen 2015; Jammes 2014; Karpukhin et al. 2014] et l'égalité découle du théorème 1.7 pour $p$ quelconque et d'un exemple construit dans [Fraser et Schoen 2011] pour le cas $p=2$.

Toutes les bornes contenues dans la table 1 sont bien conformes à la conjecture 1.6 (comparer avec les nombres chromatiques relatifs indiqués dans la table 2, section 3).

Les majorations de multiplicité pour la première valeur propre du laplacien induisent des critères de plongement de graphes dans les surfaces. Dans le cas du spectre de Steklov, on peut en tirer des critères de plongement propre dans les 
surfaces à bord. Cet aspect sera précisé dans la section 4 (corollaire 4.2). Comme par ailleurs on sait caractériser spectralement les graphes qui admettent un plongement non entrelacé dans $\mathbb{R}^{3}$, on obtient, par un cheminement inattendu, le critère de plongement qui suit. Rappelons qu'un plongement d'un graphe dans $\mathbb{R}^{3}$ est non entrelacé si tout ensemble de cycles disjoints forme un entrelacs trivial.

Corollaire 1.23. Si un graphe admet un plongement propre dans $\mathbb{M}^{2}$ ou $\mathbb{P}_{2}^{2}$, alors il admet un plongement non entrelacé dans $\mathbb{R}^{3}$.

Remarque 1.24. L'hypothèse que les sommets du graphe soient sur le bord est indispensable ; sans elle on peut trouver facilement des contre-exemples, à commencer par le graphe complet à 6 sommets.

Remarque 1.25. Si la conjecture 1.6 est vraie pour $\mathbb{K}_{1}^{2}$, alors le corollaire 1.23 s'applique aussi à cette surface.

On discutera dans la section 4 (remarque 4.8) l'existence de démonstrations plus élémentaires de ce corollaire.

Dans la section 2A, nous rappellerons quelques résultats techniques concernant le spectre de Steklov et les opérateurs sur les graphes. La section 3 sera consacrée à l'étude du nombre chromatique relatif : on justifiera en particulier cette dénomination et on calculera sa valeur sur les surfaces de petit genre. On verra dans la section 4 comment construire des valeurs propres multiples sur une surface à l'aide d'un graphe proprement plongé et on en déduira le théorème 1.7 et le corollaire 1.23. Enfin, on démontrera dans la section 5 les différentes bornes sur la multiplicité.

\section{Rappels}

2A. Le spectre de Steklov. On va rappeler ici quelques propriétés du spectre de Steklov que nous utiliserons. Il est l'ensemble des réels $\sigma$ pour lesquels le problème

$$
\begin{cases}\operatorname{div}(\gamma \nabla f)=0 & \text { dans } M, \\ \gamma \frac{\partial f}{\partial v}=\sigma \rho f & \text { sur } \partial M,\end{cases}
$$

où $v$ est un vecteur unitaire sortant normal au bord, admet des solutions non triviales. Les variétés que nous considérerons auront un bord $C^{1}$ par morceaux, ce qui est suffisant pour que le problème soit bien défini.

Le spectre de Steklov $\left(\sigma_{k}(M, g, \gamma, \rho)\right)_{k}$ est le spectre d'un opérateur DirichletNeumann $H^{1}(\partial M) \rightarrow L^{2}(\partial M)$ défini par $\Lambda_{\rho, \gamma} u=\frac{\gamma}{\rho} \frac{\partial \mathcal{H}_{\gamma} u}{\partial v}$, où $\mathcal{H}_{\gamma} u$ est le prolongement harmonique de $u$ pour la densité $\gamma$, c'est-à-dire que $\operatorname{div}\left(\gamma \nabla\left(\mathcal{H}_{\gamma} u\right)\right)=0$. Il est auto-adjoint pour la norme de Hilbert $\|u\|^{2}=\int_{\partial M} u^{2} \rho \mathrm{d} v_{g}$ (voir [Bandle 1980; Sylvester et Uhlmann 1990; Uhlmann 2009]; ces références traitent les cas $\rho \equiv 1$ ou $\gamma \equiv 1$ mais l'adaptation au cas général est aisée). 
Pour montrer le théorème 1.7 on utilisera la caractérisation variationnelle suivante du spectre :

$$
\sigma_{k}(M, g, \rho)=\inf _{V_{k+1} \in H^{1}(M)} \sup _{f \in V_{k+1} \backslash\{0\}} \frac{\int_{M}|\mathrm{~d} f|^{2} \gamma \mathrm{d} v_{g}}{\int_{\partial M} f^{2} \rho \mathrm{d} v_{g}},
$$

où $V_{k}$ parcours les sous-espaces de dimension $k$ de l'espace de Sobolev $H^{1}(M)$.

On aura aussi recours à un problème de Steklov avec condition de Neumann sur une partie du bord. Si on partitionne $\partial M$ en deux domaines (ou unions finies de domaines) $\partial_{S} M$ et $\partial_{N} M$, ce problème consiste à considérer la variante suivante du problème de Steklov :

$$
\begin{cases}\operatorname{div}(\gamma \nabla f)=0 & \text { dans } M, \\ \gamma \frac{\partial f}{\partial v}=\sigma \rho f & \text { sur } \partial M_{S}, \\ \frac{\partial f}{\partial v}=0 & \text { sur } \partial M_{N},\end{cases}
$$

c'est-à-dire qu'on demande à la fonction harmonique $f$ de vérifier la condition de Neumann sur $\partial M_{N}$. Cette condition revient à poser $\rho \equiv 0$ sur $\partial M_{N}$. Le spectre obtenu est celui d'un opérateur Dirichlet-Neumann défini sur $\partial_{S} M$. On notera $\left(\sigma_{k}\left(M, \partial M_{S}, g, \gamma, \rho\right)\right)_{k}$ son spectre.

2B. Opérateurs sur les graphes. On rappelle ici la définition des laplaciens et des opérateurs de Schrödinger sur les graphes, en se référant par exemple à [Colin de Verdière 1998].

Soit $\Gamma$ un graphe fini et $S$ son ensemble de sommets. La géométrie du graphe est déterminée par la donnée, pour chaque arête $a$ reliant deux sommets $x$ et $y$, d'un réel $l_{a}$ qui représente la longueur de cette arête. L'ensemble des $l_{a}$ sera appelé une métrique sur le graphe $\Gamma$. Le laplacien agissant sur les fonctions $f: S \rightarrow S$ est alors défini par

$$
\Delta f(x)=\sum_{a \sim x} \frac{f(x)-f\left(y_{a}\right)}{l_{a}},
$$

où la somme porte sur l'ensemble des arêtes d'extrémité $x$ et où $y_{a}$ désigne l'autre extrémité de $a$. Cet opérateur a un spectre positif, sa plus petite valeur propre est simple et vaut 0 .

Si on se donne une fonction $V$ sur l'ensemble des sommets, on peut définir un opérateur de Schrödinger $H_{V}$ sur $\Gamma$ par $H_{V} f(x)=\Delta f(x)+V(x) f(x)$. Une propriété de ces opérateurs nous sera utile : si la première valeur propre de $H_{V}$ est nulle, alors son spectre est celui de la forme quadratique d'un laplacien relativement à une norme de Hilbert $|f|=\sum_{x \in S} \mu_{x} f^{2}(x)$. Les coefficients $\mu_{x}$ s'interprètent comme une mesure sur l'espace des sommets du graphe. 


\section{Nombre chromatique relatif d'une surface à bord}

On va montrer dans cette section différents résultats concernant le nombre chromatique relatif des surfaces à bord, et en particulier calculer cet invariant sur les surfaces de petit genre.

Rappelons d'abord que le nombre chromatique d'un surface close $\Sigma$, c'est-à-dire la borne supérieure des nombres chromatiques des graphes qu'on peut plonger dans $\Sigma$, est donné par la formule

$$
\operatorname{Chr}(\Sigma)=\left\lfloor\frac{7+\sqrt{49-24 \chi(\Sigma)}}{2}\right\rfloor,
$$

sauf pour la bouteille de Klein, pour laquelle $\operatorname{Chr}\left(\mathbb{K}^{2}\right)=6$. L'étude de cet invariant, amorcée par P. J. Heawood [1890] et L. Heffter [1891] a été achevée par G. Ringel et J. Youngs [1968] en genre non nul, et K. Appel et W. Haken [1976] pour la sphère. On peut consulter [Ringel et Youngs 1968] pour un survol historique.

Notre premier résultat sur le nombre chromatique relatif est un encadrement analogue à la majoration du nombre chromatique obtenue par Heawood [1890]. Rappelons que si $\Sigma$ est une surface close, on note $\Sigma_{p}$ la surface obtenue en lui enlevant $p$ disques disjoints.

Théorème 3.2. Le nombre chromatique relatif $\mathrm{Chr}_{0}\left(\Sigma_{p}\right)$ possède les propriétés suivantes:

(a) $\operatorname{Chr}_{0}\left(\Sigma_{p}\right)$ est une fonction croissante de $p$ et vérifie les inégalités

$$
\operatorname{Chr}(\Sigma)-1 \leq \operatorname{Chr}_{0}\left(\Sigma_{p}\right) \leq \inf \left(\operatorname{Chr}(\Sigma), \frac{5+\sqrt{25-24 \chi(\Sigma)+24 p}}{2}\right) .
$$

(b) $\operatorname{Chr}_{0}\left(\Sigma_{p}\right)$ est le nombre de sommets du plus grand graphe complet proprement plongeable dans $\Sigma_{p}$.

(c) $\operatorname{Chr}_{0}\left(\Sigma_{1}\right)=\operatorname{Chr}(\Sigma)-1$ et $\operatorname{Chr}_{0}\left(\Sigma_{p}\right)=\operatorname{Chr}(\Sigma)$ si $p \geq(\operatorname{Chr}(\Sigma)-1) / 2$.

Remarque 3.4. Le fait que $\operatorname{Chr}_{0}\left(\Sigma_{p}\right)$ soit réalisé par un graphe complet établit l'équivalence entre la conjecture 1.6 et la conjecture énoncée dans [Jammes 2014].

Ce théorème permet de calculer la valeur exacte du nombre chromatique relatif sur un certain nombre de surfaces. On va compléter cette liste par des surfaces de petit genre. D'abord dans le cas où la caractéristique d'Euler de $\Sigma$ vérifie $\chi(\Sigma) \geq-1$. On aura alors la valeur de $\operatorname{Chr}_{0}\left(\Sigma_{p}\right)$ pour tout $p$ :

Proposition 3.5. $\mathrm{Chr}_{0}\left(\mathbb{P}_{2}^{2}\right)=5, \mathrm{Chr}_{0}\left(\mathbb{K}_{2}^{2}\right)=6$ et $\mathrm{Chr}_{0}\left(\# 3 \mathbb{P}_{2}^{2}\right)=7$.

On va aussi calculer quelques nombres chromatiques relatifs supplémentaires dans le cas où $-2 \geq \chi(\Sigma) \geq-7$. La liste ne sera pas exhaustive mais elle contiendra tous les cas pour lesquels on sait démontrer la conjecture 1.6 : 


\begin{tabular}{|c|c|c|c|c|c|c|c|c|c|c|c|}
\hline$p=$ & 1 & 2 & 3 & 4 & 5 & $p=$ & 1 & 2 & 3 & 4 & 5 \\
\hline $\mathbb{S}^{2}$ & 3 & 4 & 4 & 4 & 4 & $\# 4 \mathbb{P}^{2}$ & 7 & ? & 8 & 8 & 8 \\
\hline $\mathbb{P}^{2}$ & 5 & 5 & 6 & 6 & 6 & $\chi=-3$ & 8 & 8 & 9 & 9 & 9 \\
\hline $\mathbb{K}^{2}$ & 5 & 6 & 6 & 6 & 6 & $\chi=-4$ & 8 & ? & 9 & 9 & 9 \\
\hline $\mathbb{T}^{2}$ & 6 & 6 & 7 & 7 & 7 & $\chi=-5$ & 9 & 9 & 9 & 10 & 10 \\
\hline$\chi=-1$ & 6 & 7 & 7 & 7 & 7 & $\# 4 \mathbb{T}^{2}$ & 9 & 9 & 10 & 10 & 10 \\
\hline$\# 2 \mathbb{T}^{2}$ & 7 & 8 & 8 & 8 & 8 & $\chi=-7$ & 9 & ? & 10 & 10 & 10 \\
\hline
\end{tabular}

Table 2. Nombre chromatique relatif des surfaces de petit genre.

Proposition 3.6. Soit $\Sigma$ une surface close.

(1) $\mathrm{Chr}_{0}\left(\# 2 \mathbb{\varpi}_{2}^{2}\right)=\mathrm{Chr}_{0}\left(\# 4 \mathbb{P}_{3}^{2}\right)=8$.

(2) Si $\chi(\Sigma)=-3$ ou -4 , alors $\operatorname{Chr}_{0}\left(\Sigma_{3}\right)=9$.

(3) Si $\chi(\Sigma)=-5$ alors $\operatorname{Chr}_{0}\left(\Sigma_{4}\right)=10$.

(4) $\operatorname{Chr}_{0}\left(\# 4 \mathbb{\Psi}_{3}^{2}\right)=10$.

La table 2 rassemble en fonction de $\Sigma$ et $p$ les nombres chromatiques qu'on peut calculer à l'aide du théorème 3.2, des propositions 3.5 et 3.6 et en utilisant la monotonie du nombre chromatique relatif par rapport à $p$ et par somme connexe. On se limite aux surfaces $\Sigma$ telles que $\chi(\Sigma) \geq-7$.

On va montrer séparément le résultat le plus technique du théorème 3.2 :

Lemme 3.7.

$$
\operatorname{Chr}_{0}\left(\Sigma_{p}\right) \leq \frac{5+\sqrt{25-24 \chi(\Sigma)+24 p}}{2} .
$$

Démonstration. On pose $c_{p}=\lfloor(5+\sqrt{25-24 \chi(\Sigma)+24 p}) / 2\rfloor$. Étant donné un graphe $\Gamma$ plongé proprement dans $\Sigma_{p}$, on va montrer qu'on peut le colorier avec $c_{p}$ couleurs. Quitte à ajouter (temporairement) des sommets et des arêtes, on peut supposer d'une part que toutes les faces de la décomposition de $\Sigma_{p}$ induite par $\Gamma$ sont simplement connexes, et d'autre part que $\partial \Sigma_{p}$ est recouvert par des arêtes. Il peut apparaître des arêtes multiples (plusieurs arêtes ayant les mêmes sommets) ou des boucles (arête reliant un sommet à lui-même) lors de cette étape mais ça n'est pas gênant pour la suite. On note $s$ et $a$ le nombre de sommets et d'arêtes de $\Gamma, \delta$ le degré minimal de ses sommets et $f$ le nombre de faces de la décomposition de $\Sigma_{p}$ induite par $\Gamma$.

On commence par majorer $\delta$. Comme chaque face est bordée par au moins trois arêtes et que chaque arête (sauf celles qui sont sur le bord, qui sont en nombre égal au nombre de sommets) est adjacente à deux faces, on a $3 f \leq 2 a-s$. De plus, le degré minimal vérifie les inégalités $\delta s \leq 2 a$ et $\delta+1 \leq s$. Il découle alors de la formule d'Euler-Poincaré, en notant $\chi=\chi(\Sigma)-p$ la caractéristique d'Euler de 
$\Sigma_{p}$ et en supposant que $\delta \geq 4$, que

$$
\begin{aligned}
6 \chi & =6 f+6 s-6 a \leq 4 s-2 a \\
& \leq 4 s-\delta s=(4-\delta) s \leq(4-\delta)(\delta+1) \\
& \leq-\delta^{2}+3 \delta+4
\end{aligned}
$$

Comme $\delta$ vérifie l'inéquation $\delta^{2}-3 \delta-4+6 \chi \leq 0$, on en déduit que

$$
\delta \leq\lfloor(3+\sqrt{25-24 \chi}) / 2\rfloor=c_{p}-1 .
$$

Si $\delta \leq 4$, cette inégalité est trivialement vérifiée dès que $\chi \leq 0$.

On supprime ici les boucles qui sont apparues en ajoutant des arêtes dans l'étape précédente ; la majoration de $\delta$ reste valide.

On construit ensuite un coloriage par une double récurrence sur $p$, et sur $s$ à $p$ fixé. Dans la récurrence sur $s$, on aura besoin que la majoration de $\delta$ s'applique bien à tous les graphes considérés.

Si $s \leq c_{p}$, le graphe $\Gamma$ se colorie évidemment avec $c_{p}$ couleurs. Dans le cas contraire, on note $x$ un sommet de degré minimal et on considère le graphe $\Gamma^{\prime}$ obtenu en supprimant le sommet $x$ et les arêtes qui lui sont adjacentes. La récurrence se décompose en deux cas, selon que la composante de bord où est situé $x$ porte d'autres sommets de $\Gamma$ où non. Soulignons que si $p=1$, tous les sommets sont sur la même composante de bord donc seul le premier cas se présentera.

Dans le premier cas, on ajoute une arête qui joint les deux voisins de $x$ sur la même composante de bord (s'il ne reste qu'un seul point sur cette composante, l'arête supplémentaire forme une boucle) et on appelle encore $\Gamma^{\prime}$ le graphe obtenu. La démonstration de la majoration $\delta \leq c_{p}-1$ obtenue pour $\Gamma$ s'applique alors aussi à $\Gamma^{\prime}$. Comme $\Gamma^{\prime}$ a $s-1$ sommets, on peut lui appliquer l'hypothèse de récurrence et le colorier avec $c_{p}$ couleurs. On applique le même coloriage à tous les sommets de $\Gamma$ sauf $x$, et comme le degré $\delta$ de $x$ est majoré par $c_{p}-1$ on peut colorier $x$ avec une couleur différente de ses voisins.

Dans le second cas, la composante de bord de $x$ ne porte aucun sommet de $\Gamma^{\prime}$ et on se ramène à la surface $\Sigma_{p-1}$ en collant un disque le long de cette composante de bord. Le graphe $\Gamma^{\prime}$ est proprement plongé dans $\Sigma_{p-1}$, donc admet un coloriage à $c_{p-1}$ couleurs par hypothèse de récurrence. Comme la constante $c_{p}$ croît avec $p$ il admet un coloriage à $c_{p}$ couleurs. On conclut comme dans le cas précédent.

Démonstration du théorème 3.2. On va noter temporairement $\kappa\left(\Sigma_{p}\right)$ le nombre de sommets du plus grand graphe complet proprement plongeable dans $\Sigma_{p}$. Il est clair qu'un plongement dans $\Sigma_{p}$ induit un plongement dans $\Sigma_{p+1}$ et dans $\Sigma$, donc $\kappa\left(\Sigma_{p}\right)$ et $\operatorname{Chr}_{0}\left(\Sigma_{p}\right)$ sont des fonctions croissantes de $p$ et

$$
\kappa\left(\Sigma_{p}\right) \leq \operatorname{Chr}_{0}\left(\Sigma_{p}\right) \leq \operatorname{Chr}(\Sigma) .
$$


Supposons que $p=1$. Si on se donne un plongement propre du graphe complet $K_{n}$ à $n$ sommets dans $\Sigma_{1}$, on peut construire un plongement de $K_{n+1}$ dans $\Sigma$ en collant un disque sur le bord de $\Sigma_{1}$, en ajoutant un sommet sur ce disque et en le reliant par des arêtes aux sommets de $K_{n}$ (c'est possible puisqu'ils sont tous sur le bord du disque). Réciproquement, étant donné un graphe complet $K_{n+1}$ étant plongé dans $\Sigma$, on peut enlever un disque contenant un seul sommet et déplacer les autres sommets de manière à ce qu'ils se situent sur le bord du disque. On obtient un plongement de $K_{n}$ dans $\Sigma_{1}$ dont les sommets sont sur $\partial \Sigma_{1}$. On en déduit que $\kappa\left(\Sigma_{1}\right)=\operatorname{Chr}(\Sigma)-1$ et donc que

$$
\operatorname{Chr}(\Sigma)-1 \leq \kappa\left(\Sigma_{p}\right) \leq \operatorname{Chr}_{0}\left(\Sigma_{p}\right) \leq \operatorname{Chr}(\Sigma),
$$

ce qui, avec le lemme 3.7, conclut la démonstration du point (a) du théorème.

On peut maintenant montrer le point (b), c'est-à-dire que $\kappa\left(\Sigma_{p}\right)=\operatorname{Chr}_{0}\left(\Sigma_{p}\right)$. Ces deux nombres ne peuvent prendre que les valeurs $\operatorname{Chr}(\Sigma)-1$ et $\operatorname{Chr}(\Sigma)$. Si $\operatorname{Chr}_{0}\left(\Sigma_{p}\right)=\operatorname{Chr}(\Sigma)-1$, alors on a immédiatement l'égalité dans les deux premières inégalités de (3.10). Supposons donc que $\operatorname{Chr}_{0}\left(\Sigma_{p}\right)=\operatorname{Chr}(\Sigma)$ et considérons un graphe $\Gamma$ proprement plongé dans $\Sigma_{p}$, dont le nombre chromatique est $\operatorname{Chr}(\Sigma)$ et qui est critique, c'est-à-dire qu' on ne peut pas lui enlever d'arêtes sans diminuer son nombre chromatique. Cette propriété de criticité est intrinsèque au graphe $\Gamma$, donc il est aussi critique comme graphe plongé dans $\Sigma$. Or, on sait qu'un tel graphe est nécessairement un graphe complet (cf. [Bollobás 1998, Chapter V, p. 156-157]), donc on a égalité dans l'inégalité (3.9).

On a déjà montré que $\operatorname{Chr}_{0}\left(\Sigma_{1}\right)=\kappa\left(\Sigma_{1}\right)=\operatorname{Chr}(\Sigma)-1$. Il reste à montrer que $\operatorname{Chr}_{0}\left(\Sigma_{p}\right)=\operatorname{Chr}(\Sigma)$ si $p \geq(\operatorname{Chr}(\Sigma)-1) / 2$ pour conclure le point (c) du théorème. On considère le graphe complet à $\operatorname{Chr}(\Sigma)$ sommets qu'on plonge dans $\Sigma$ et on construit un plongement de ce graphe dans $\Sigma_{p}$ en enlevant $p$ disques (pour $p$ suffisamment grand) à $\Sigma$ de manière à ce que les disques ne rencontrent pas les arêtes et que les sommets soient situés sur le bord des disques. Pour déterminer une valeur de $p$ adéquate, on remarque qu'en plaçant le premier disque de manière quelconque, on peut placer (au moins) trois sommets du graphe sur son bord. On peut ensuite regrouper les autres sommets par deux et placer un disque adjacent à ces deux sommets et qui longe l'arête qui les relie. En fonction de la parité du nombre de sommets, il peut en rester un qui nécessite un disque supplémentaire. Au total, on a utilisé $\lceil(\operatorname{Chr}(\Sigma)-1) / 2\rceil$ disques.

Démonstration de la proposition 3.5. Le fait que $\operatorname{Chr}_{0}\left(\mathbb{P}_{2}^{2}\right)=5$ découle des théorèmes 1.7 et 1.20 .

Le calcul de $\operatorname{Chr}_{0}\left(\mathbb{K}_{2}^{2}\right)$ repose sur une amélioration de l'estimation faite par le théorème 3.2 dans le cas où $p \geq(\operatorname{Chr}(\Sigma)-1) / 2$ en utilisant le plongement explicite d'un graphe complet dans la surface. Dans le cas de la bouteille de Klein, 


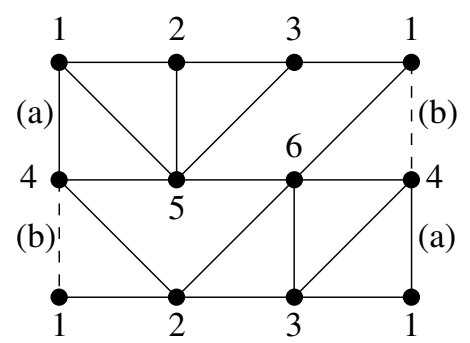

Figure 1. Plongement de $K_{6}$ dans $\mathbb{K}^{2}$.

le plongement du graphe complet à 6 sommets est représenté sur la figure 1 (les lettres indiquent comment sont identifiés les cotés droite et gauche).

En enlevant les faces (125) et (346), on obtient un plongement propre de $K_{6}$ dans $\mathbb{K}_{2}^{2}$.

Il reste à calculer $\mathrm{Chr}_{0}\left(\# 3 \mathbb{P}_{2}^{2}\right)$. On sait déjà que $\mathrm{Chr}_{0}\left(\# 3 \mathbb{P}_{2}^{2}\right) \leq 7$ (théorème 3.2), il suffit donc d'exhiber un plongement propre de $K_{7}$. On procède de la manière suivante : topologiquement, on enlève deux disques au ruban de Möbius $\mathbb{M}^{2}$ et on colle un pantalon (une sphère privée de trois disques) sur les deux composantes de bord ainsi crées. Pour construire le graphe, on part du graphe $K_{5}$ plongé dans le ruban de Möbius et on ajoute deux sommets situés sur le troisième bord du pantalon. Les détails de la construction sont représentés sur la figure 2.

En haut de la figure est représenté le graphe $K_{5}$ plongé dans $\mathbb{M}^{2}$. On enlève deux disques situés dans les faces (123) et (451). En bas est représenté le pantalon, les deux bords intérieurs étant recollés sur $\mathbb{M}^{2}$. Les sommets 6 et 7 sont bien reliés entre eux et aux cinq premiers sommets.

Démonstration de la proposition 3.6. Pour calculer les nombres chromatiques traités ici, on procède comme on l'a fait pour $\mathrm{Chr}_{0}\left(\mathbb{K}_{2}^{2}\right)$ : on considère le découpage de

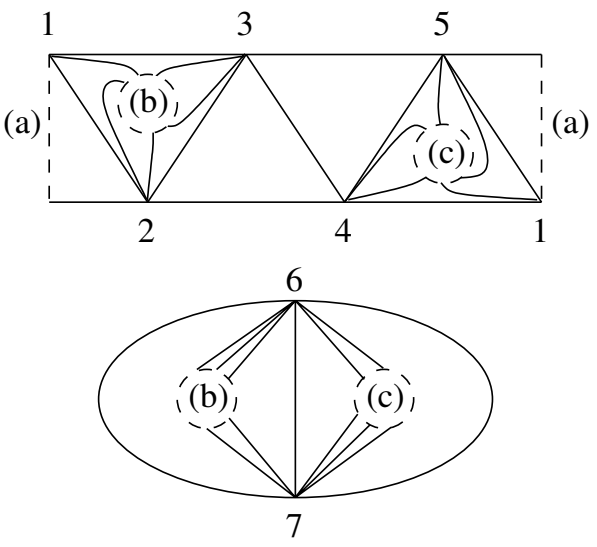

Figure 2. Plongement propre de $K_{7}$ dans $\# 3 \mathbb{P}_{2}^{2}$. 

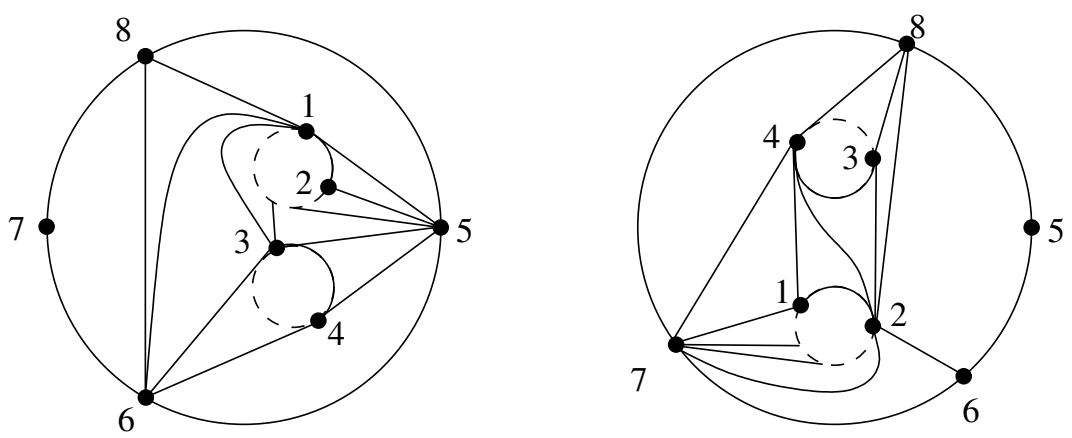

Figure 3. Plongement de $K_{8}$ dans $\mathbb{\nabla}^{2} \# \mathbb{\varpi}^{2}$.

$\Sigma$ induit par le plongement d'un graphe complet maximal et on cherche comment enlever des disques de manière à placer tous les sommets sur le bord.

On commence par le cas $\chi=-2$. Des plongements de $K_{8}$ dans $\mathbb{T}^{2} \# \mathbb{T}^{2}$ et $\# 4 \mathbb{P}_{2}^{2}$ ont été décrits respectivement par L. Heffter [1891] et I. N. Kagno [1935]. La figure 3 représente un plongement de $K_{8}$ dans $\mathbb{T}^{2} \# \mathbb{T}^{2}$ : la surface $\mathbb{T}^{2} \# \mathbb{T}^{2}$ est obtenue en recollant les deux pantalons représentés sur la figure le long de leurs bords, la position des sommets de $K_{8}$ indiquant l'orientation du recollement. On peut remarquer que l'arête (34) peut être placée arbitrairement d'un coté ou de l'autre du bord des pantalons portant les deux sommets. En fonction de ce choix, on obtient une surface orientable ou non.

On peut noter que la décomposition de la surface induite par le plongement comporte un quadrilatère (5628). Si on enlève cette face, on peut ensuite enlever deux autres disques, par exemple le long des arêtes (13) et (47), de manière à obtenir un plongement propre de $K_{8}$ dans $\left(\mathbb{T}^{2} \# \mathbb{T}^{2}\right)_{3}$ ou $\left(\# 4 \mathbb{P}^{2}\right)_{3}$ selon le choix d'orientation. Par conséquent, $\mathrm{Chr}_{0}\left(\left(\mathbb{T}^{2} \# \mathbb{T}^{2}\right)_{3}\right)=\mathrm{Chr}_{0}\left(\# 4 \mathbb{P}_{3}^{2}\right)=8$.

La figure 3 ne permet pas de montrer que $\operatorname{Chr}_{0}\left(\left(\mathbb{T}^{2} \# \mathbb{T}^{2}\right)_{2}\right)=8$ car les huit sommets ne sont pas sur le bord de deux quadrilatères. Cependant, le plongement de $K_{8}$ dans $\left(\mathbb{T}^{2} \# \mathbb{T}^{2}\right)_{2}$ décrit dans [Ringel 1974, p. 23, Table (2.9)] vérifie cette propriété, les deux quadrilatères étant (0246) et (1357).

Le plongement de $K_{9}$ dans $\# 5 \mathbb{P}^{2}$ a été construit par H.S.M. Coxeter [1943]. Ce plongement triangule la surface et on peut vérifier qu'on peut trouver trois faces dont les sommets sont tous distincts (par exemple les faces (129), (678) et (345) de la figure 11 de [Coxeter 1943]). En enlevant ces faces, on obtient un plongement propre de $K_{9}$ dans $\# 5 \mathbb{P}_{3}^{2}$.

Le plongement de $K_{9}$ dans $\# 3 \mathbb{T}^{2}$ exhibé dans [Heffter 1891] comporte une face hexagonale ayant 5 sommets distincts. En enlevant cette face et deux autres disques, on peut donc obtenir un plongement propre de $K_{9}$ dans $\# 3 \mathbb{\square}_{3}^{2}$. Le cas de $\# 6 \mathbb{P}_{3}^{2}$ s'obtient par somme connexe de $\# 5 \mathbb{P}_{3}^{2}$ avec $\mathbb{P}^{2}$. 
R. C. Bose [1939] a donné la construction d'un plongement de $K_{10}$ dans $\# 7 \mathbb{P}_{3}^{2}$, elle est aussi étudiée (et illustrée par une figure) par Coxeter [1943]. Le graphe triangule la surface et on peut trouver deux triangles n'ayant pas de sommets communs (voir la figure 14 de [Coxeter 1943]). Les quatre autres sommets du graphe peuvent se placer sur le bord de deux disques; en enlevant quatre disques, on peut donc bien placer tous les sommets sur le bord.

Le plongement de $K_{10}$ dans \#4T⿱乛龰 ${ }^{2}$ exhibé dans [Heffter 1891] comporte des quadrilatères, chacun ayant ses quatre sommets distincts. Après avoir enlevé un de ces quadrilatères, il reste 6 sommets à placer sur un bord, ce qu' on peut faire en enlevant deux autres faces triangulaires (les trois faces sont $(2,1,10,4),(3,6,8)$, $(1,4,5)$ dans la construction de Heffter).

\section{Construction de valeurs propres multiples}

Cette section est consacrée à la démonstration du théorème 1.7. Elle est très similaire à la démonstration du théorème 1.2 de [Jammes 2014]. On va donc rappeler les différentes étapes en indiquant les modifications à apporter. Le principe consiste à faire tendre le début du spectre de la surface vers celui d'un laplacien combinatoire d'un graphe complet, puis d'utiliser la propriété de stabilité du spectre de ce graphe (cf. [Colin de Verdière 1988] les rappels ci dessous) pour exhiber une valeur propre multiple sur la surface.

Par cette méthode on va en fait montrer un résultat analogue pour un graphe fini quelconque, cela nous sera utile en particulier pour démontrer le corollaire 1.23. L'énoncé général fait intervenir l'invariant de graphe forgé par Colin de Verdière [1990] (voir aussi [Colin de Verdière 1998]). Rappelons que cet invariant, qu'on notera $\mu(\Gamma)$, peut se définir comme étant la multiplicité maximale de la deuxième valeur propre des opérateurs de Schrödinger combinatoires sur le graphe $\Gamma$ pour lesquels cette valeur propre multiple vérifie l'hypothèse de transversalité d'Arnol'd formalisée dans [Colin de Verdière 1988] (on dit alors que la multiplicité est stable). Si on note $M$ la matrice de cet opérateur de Schrödinger, cette hypothèse de tranvsersalité consiste en ce que dans l'espace des matrices symétriques, le sous-espace des matrices d'opérateurs de Schrödinger et le sous-espace des matrices possédant une deuxième valeur propre de même multiplicité se coupent transversalement. En pratique, on manipulera des laplaciens à densité sur le graphe plutôt que des opérateurs de Schrödinger, les deux points de vue étant équivalents (cf. section 2B). On peut consulter [Colin de Verdière 1988; 1998] ou [Jammes 2009] pour une description plus détaillée de cette propriété de transversalité et de son utilisation.

Théorème 4.1. Soit $\Gamma$ un graphe admettant un plongement propre dans une surface compacte à bord $\Sigma$. Il existe des densités $\gamma \in C^{\infty}(\Sigma), \rho \in C^{\infty}(\partial \Sigma)$ et une métrique $g$ sur $\Sigma$ telles que la multiplicité de $\sigma_{1}(\Sigma, g, \rho, \gamma)$ égale $\mu(\Gamma)$. 
Le théorème 1.7 s'en déduit en remarquant d'une part qu'un graphe complet à $n$ sommets vérifie $\mu\left(K_{n}\right)=n-1$ [Colin de Verdière 1988, section 4, théorème 1], et d'autre part que si $n=\mathrm{Chr}_{0}(\Sigma)$, alors $K_{n}$ admet un plongement propre dans $\Sigma$ (théorème 3.2 de la section précédente).

On peut aussi en déduire le critère de plongement qui suit :

Corollaire 4.2. Si $\Gamma$ admet un plongement propre dans $\Sigma$, alors $\mu(\Gamma) \leq m_{1}(\Sigma)$.

Le corollaire 1.23 est une conséquence de ce dernier; il sera traité à la section 4A.

La première étape de la démonstration du théorème 4.1 consiste à construire une variété dont le début du spectre de Steklov tend vers celui d'un laplacien combinatoire pour une métrique donnée sur $\Gamma$, cette variété pouvant s'interpréter comme un voisinage tubulaire de $\Gamma$. En dimension 2, cette variété, qu'on notera $\Omega$, est formée de $n$ demi-disques ( $n$ étant le nombre de sommets de $\Gamma$ ), deux demidisques étant reliés par un rectangle fin si les sommets correspondants sont reliés par une arête. Sur ce domaine, on considère le spectre de Steklov-Neumann (voir les rappels de la section 2A) : le bord de Steklov est formé des diamètres des demi-disques, et on pose la condition de Neumann sur le reste du bord.

Lemme 4.3 [Jammes 2014, théorème 4.3]. Il existe une famille de métriques $g_{\varepsilon}$ sur $\Omega$ telle que les $n$ premières valeurs propre de Steklov de $\left(\Omega, g_{\varepsilon}\right)$ tendent vers le spectre de $\Delta_{\Gamma}$, avec convergence des espaces propres.

Remarque 4.4. Dans [Jammes 2014], ce théorème est démontré pour des densités uniformes sur $\partial \Omega_{S}$ et sur les sommets du graphes. Si on munit les sommets du graphe d'une autre mesure, on peut adapter la démonstration en munissant chaque composante de $\partial \Omega_{S}$ (les diamètres des demi-disques) de la densité correspondante. On peut donc faire tendre le spectre de $\Omega$ non seulement vers le spectre d'un laplacien sur $\Gamma$, mais aussi d'un opérateur de Schrödinger.

Le deuxième ingrédient de la démonstration est un résultat de convergence du spectre d'une surface vers celui d'un de ses domaines. Sur un domaine $U$ d'une surface à bord $\Sigma$, on considérera le problème de Steklov-Neumann avec $\partial U_{S}=\partial U \cap \partial \Sigma$ comme bord de Steklov et $\partial U_{N}=\partial U \backslash \partial U_{S}$ comme bord de Neumann :

Lemme 4.5. Soit $(\Sigma, g)$ une surface riemannienne compacte à bord, $\rho$ une densité sur $\partial \Sigma$ et $U$ un domaine de $\Sigma$ à bord $C^{1}$ par morceaux tel que $\partial U_{S}=\partial U \cap \partial \Sigma$ soit non vide. Il existe des familles de densités $\gamma_{\varepsilon} \in C^{\infty}(\Sigma)$ et de métriques $g_{\varepsilon}$ telles que $\sigma_{k}\left(\Sigma, g_{\varepsilon}, \gamma_{\varepsilon}, \rho\right) \rightarrow \sigma_{k}\left(U, \partial U_{S}, g, \rho_{\mid \partial U_{S}}\right)$ avec convergence des espaces propres quand $\varepsilon$ tend vers 0 .

La démonstration est la même que celle du théorème 3.8 de [Jammes 2014], avec l'adaptation à la dimension 2 introduite par Colin de Verdière [1987]. On introduit une densité singulière $\bar{\gamma}_{\eta}$ qui vaut 1 sur $U$ et $\eta$ sur $\Sigma \backslash U$, une métrique $\bar{g}_{\eta}$ égale à 
$g$ sur $U$ et à $\eta^{2} g$ sur $\Sigma \backslash U$ et on travaille avec les familles de formes quadratiques et de normes de Hilbert induites par $\bar{\gamma}_{\eta}$ et $\bar{g}_{\eta}$ :

$$
Q_{\eta}(f)=\inf _{\tilde{f}_{\mid \partial M}=f}\left(\int_{U}|\mathrm{~d} \tilde{f}|^{2} \mathrm{~d} v_{g}+\eta^{3} \int_{M \backslash U}|\mathrm{~d} \tilde{f}|^{2} \mathrm{~d} v_{g}\right),
$$

et $|f|_{\eta}=\int_{\partial U_{S}} f^{2} \mathrm{~d} v_{g}+\eta^{2} \int_{\partial M \backslash \partial U_{S}} f^{2} \mathrm{~d} v_{g}$. Le reste de la démonstration (convergence de spectre et lissage de la densité et de la métrique) est identique à [Jammes 2014].

Démonstration du théorème 4.1. Soit $\Gamma$ admettant un plongement propre dans $\Sigma$. On peut trouver un voisinage de $\Gamma$ dans $\Sigma$ difféomorphe au domaine $\Omega$ décrit précédemment. Étant donnée une métrique et une mesure sur $\Gamma$, on peut trouver une famille de métriques $g_{\varepsilon}$ sur $\Omega$ dont le début du spectre de Steklov-Neumann tend vers le spectre du laplacien combinatoire sur $\Gamma$ (lemme 4.3). Pour toute métrique sur $\Omega$ on peut trouver une famille de densités sur $\Sigma$ et $\partial \Sigma$ telle que le spectre de Steklov de $\Sigma$ tende vers celui de $\Omega$ (lemme 4.5). Par conséquent, il existe des familles de métriques $g_{\varepsilon}$ de densités $\rho_{\varepsilon}$ (étendant la métrique $g_{\varepsilon}$ et la densité $\rho$ de $\Omega)$ et de densités $\gamma_{\varepsilon}$ sur $\Sigma$ telles que le début du spectre de $\left(\Sigma, g_{\varepsilon}, \rho_{\varepsilon}, \gamma_{\varepsilon}\right)$ tende vers le spectre du laplacien combinatoire sur $\Gamma$.

En munissant $\Gamma$ de la métrique et de la mesure réalisant la multiplicité $\mu(\Gamma)$, on peut faire tendre le spectre de $\Sigma$ vers un spectre limite (la métrique et la densité étant alors dégénérées) ayant la multiplicité souhaitée. Pour que $\sigma_{1}\left(\Sigma, g_{\varepsilon}, \rho_{\varepsilon}, \gamma_{\varepsilon}\right)$ soit de multiplicité $\mu(\Gamma)$ pour une métrique $g_{\varepsilon}$ et des densités $\rho_{\varepsilon}$ et $\gamma_{\varepsilon}$ lisses, on utilise la propriété de stabilité de la multiplicité : quitte à déformer l'opérateur sur $\Gamma$, on peut trouver un $\varepsilon>0$ tel que $\sigma_{1}\left(\Sigma, g_{\varepsilon}, \gamma_{\varepsilon}\right)$ soit de multiplicité $\mu(\Gamma)$.

4A. Critère de plongement non entrelacé. Cette section est consacrée au corollaire 1.23, dont la démonstration repose sur les propriétés de l'invariant $\mu$ des graphes défini par Colin de Verdière et déjà utilisé dans le paragraphe précédent. On utilisera en particulier le fait que cet invariant permet de caractériser les graphes non entrelacés :

Théorème 4.7 [Robertson et al. 1995; Bacher et Colin de Verdière 1995; Lovász et Schrijver 1998]. Un graphe $\Gamma$ est non entrelacé si et seulement si $\mu(\Gamma) \leq 4$.

Le fait $\mu(\Gamma) \leq 4$ implique le non entrelacement du graphe découle de la caractérisation par mineurs exclus des graphes non entrelacés due à N. Robertson, P. Seymour et R. Thomas [1995] et du calcul de $\mu$ sur les graphes de la famille de Petersen fait dans [Bacher et Colin de Verdière 1995]. La réciproque a été montrée par L. Lovász et A. Schrijver [1998].

Le corollaire 1.23 découle de la remarque suivante : sachant que $m_{1}\left(\mathbb{M}^{2}\right)=$ $m_{1}\left(\mathbb{P}_{2}^{2}\right)=4$, si $\Gamma$ admet un plongement propre dans $\mathbb{M}^{2}$ ou $\mathbb{P}_{2}^{2}$, alors $\mu(\Gamma) \leq 4$ d'après le théorème 4.1 . Le théorème 4.7 permet alors de conclure. 
Remarque 4.8. Si $\Gamma \rightarrow \Sigma$ est un plongement propre d'un graphe dans une surface et $\Sigma \rightarrow \mathbb{R}^{3}$ un plongement de cette surface dans $\mathbb{R}^{3}$, on peut espérer par composition obtenir un plongement non entrelacé de $\Gamma$. Si le plongement usuel $\mathbb{M}^{2} \rightarrow \mathbb{R}^{3}$ semble suffire pour tout plongement propre $\Gamma \rightarrow \mathbb{M}^{2}$, il n'en va pas de même pour $\mathbb{P}_{2}^{2}$ : si on considère le plongement $\mathbb{P}_{2}^{2} \rightarrow \mathbb{R}^{3}$ obtenu en enlevant un disque au plongement $\mathbb{M}^{2} \rightarrow \mathbb{R}^{3}$, il existe des plongements propres $\Gamma \rightarrow \mathbb{M}^{2}$ tels que le plongement $\Gamma \rightarrow \mathbb{R}^{3}$ induit soit entrelacé (on utilise le fait que le bord et l'âme du ruban de Möbius forment un entrelacs non trivial). Mais cela n'exclut pas l'existence, pour un plongement $\Gamma \rightarrow \mathbb{M}^{2}$ fixé, d'un autre plongement $\mathbb{P}_{2}^{2} \rightarrow \mathbb{R}^{3}$ tel que le plongement de $\Gamma$ soit non entrelacé.

\section{Bornes sur la multiplicité}

5A. Propriétés de l'ensemble nodal d'une fonction propre. Ce paragraphe sera consacré aux propriétés générales de l'ensemble nodal des fonctions propres du problème de Steklov. Il s'agit généralement de rappels de résultats montrés dans [Fraser et Schoen 2015; Jammes 2014; Karpukhin et al. 2014], ou remontant à l'étude des fonctions propres du laplacien. Certaines sont reformulées ou précisées. Elles sont regroupées en deux théorèmes : le théorème 5.1 rassemble les propriétés locales de l'ensemble nodal et le théorème 5.5 les propriétés topologiques globales. Ces résultats ont généralement été démontrés dans le cas homogène mais leur démonstration reste valide dans le cas général. Si une fonction $f$ s'annule en un point $p$, on appelle ordre d'annulation de $f$ en $p$ le plus petit entier $k$ tel que $\nabla^{k} f \neq 0$.

Théorème 5.1 [Cheng 1976; Fraser et Schoen 2015]. Soit p un point de l'ensemble nodal d'une fonction propre $f$ du problème de Steklov sur $\Sigma$. On note $k$ l'ordre d'annulation de $f$ en $p$.

Au voisinage de p l'ensemble nodal est la réunion de $k$ arcs de courbes s'intersectant en $p$, de courbure géodésique nulle en $p$ et formant un système équiangulaire. De plus :

(1) Si p est un point intérieur à $\Sigma$, alors $p$ est l'extrémité de $2 k$ arcs nodaux.

(2) Si p est sur le bord de $\Sigma$, alors $p$ est l'extrémité de $k$ arcs nodaux rencontrant transversalement le bord.

On peut en déduire, comme dans [Cheng 1976], que l'ensemble nodal est la réunion de sous-variétés de dimension 1 immergées dans $\Sigma$, chaque sous-variété étant soit un cercle ne rencontrant pas le bord, soit un intervalle dont les extrémités sont sur le bord. On appellera lignes nodales ces sous-variétés. On peut aussi interpréter l'ensemble nodal comme un graphe plongé dans $\Sigma$, les sommets étant les points critiques de la fonction propre. On appellera arêtes nodales les arêtes 
de ce graphe. Soulignons que les arêtes nodales sont plongées alors que les lignes nodales sont seulement immergées.

On aura aussi besoin du lemme qui suit et qui assure l'existence d'une fonction propre s'annulant à un ordre élevé quand la multiplicité est grande :

Lemme 5.2. Soit $k$ un entier positif, $p$ un point de $\Sigma$ et $E$ un espace propre du problème de Steklov. Si l'une des deux conditions suivantes est vérifiée

(1) $p$ est un point intérieur à $\Sigma$ et $E$ est de dimension au moins $2 k$;

(2) $p$ est sur le bord de $\Sigma$ et $E$ est de dimension au moins $k+1$;

alors $E$ contient une fonction qui s'annule à l'ordre $k$ en $p$.

Démonstration. Le cas où $p$ est intérieur à $\Sigma$ est traité dans [Besson 1980]. On va donc supposer sur $p \in \partial \Sigma$ et que $E$ est de dimension au moins $k+1$. Par une déformation conforme, on peut identifier localement la surface au demi-plan supérieur muni des coordonnées $(x, y)$ et le point $p$ au point $(0,0)$.

Comme $E$ est de dimension au moins $k+1$, on peut trouver une fonction non nulle de $E$ telle que

$$
f(p)=\frac{\partial f}{\partial x}(p)=\cdots=\frac{\partial^{k-1} f}{\partial x^{k-1}}(p)=0 .
$$

On sait aussi que $f$ vérifie l'équation aux valeurs propres $\partial f / \partial y=\sigma \rho f$ en tout point du bord. En dérivant cette relation par rapport à $x$ et en l'évaluant au point $p$, on obtient que

$$
\frac{\partial f}{\partial y}(p)=\frac{\partial^{2} f}{\partial x \partial y}(p)=\cdots=\frac{\partial^{k} f}{\partial x^{k-1} \partial y}(p)=0 .
$$

Ces relations suffisent pour conclure si $k=1$ ou 2 .

Enfin, la fonction $f$ est harmonique relativement à la densité $\gamma$, c'est-à-dire qu'elle vérifie la relation $\operatorname{div}(\gamma \nabla f)=0$. Par conséquent, $\frac{\partial^{2} f}{\partial x^{2}}+\frac{\partial^{2} f}{\partial y^{2}}$ peut s'écrire comme une expression d'ordre 0 ou 1 en $f$, et donc, en utilisant les relations déjà obtenues, $\frac{\partial^{2} f}{\partial y^{2}}(p)=0$ si $k \geq 3$. En dérivant successivement l'équation $\operatorname{div}(\gamma \nabla f)=0$, on obtient par récurrence que toutes les dérivées partielles de $f$ d'ordre au moins 2 par rapport à $y$ sont nulles en $p$ jusqu'à l'ordre souhaité.

Théorème 5.5. La décomposition nodale de $\Sigma$ vérifie les propriétés suivantes:

(1) Tous les domaines nodaux rencontrent le bord de la surface $\Sigma$.

(2) Les domaines nodaux et les composantes connexes du graphe nodal d'une fonction propre sont incompressibles dans la surface $\Sigma$.

(3) Si $\Gamma$ est l'union d'une ou plusieurs composantes connexes du graphe nodal, alors on a la relation $\chi(\Gamma) \geq \chi(\Sigma)$. 
Démonstration. Les premières propriétés énoncées dans ce théorème sont déjà connues (voir par exemple [Jammes 2014]), mais nous allons rappeler leur preuve.

Si un domaine nodal ne rencontre pas le bord de $\Sigma$, alors la fonction propre est nulle sur toute la frontière du domaine, donc elle est nulle à l'intérieur du domaine puisqu'elle est harmonique et par conséquent elle est nulle partout selon la propriété d'unique prolongement.

Supposons qu'un domaine nodal $D$ contienne une courbe contractile dans $\Sigma$ mais pas dans $D$. Alors le disque bordé par cette courbe contient un domaine nodal distinct de $D$ et qui ne rencontre pas le bord de $\Sigma$. Or on vient de voir que c'est impossible, donc toute courbe de $D$ contractile dans $\Sigma$ est contractile dans $D$. Le même argument montre que le graphe nodal est incompressible.

Enfin, si on note $\Gamma$ la réunion d'une ou plusieurs composantes connexes du graphe nodal, et $D_{i}$ les composantes connexes de $\Sigma \backslash \Gamma$, la formule d'Euler-Poincaré nous dit que

$$
\chi(\Sigma)=\chi(\Gamma)+\sum_{i} \chi\left(D_{i}\right)
$$

Pour un $i$ donné, la caractéristique d'Euler de l'intérieur de $D_{i}$ est au plus égale à 1 . Mais comme $D_{i}$ contient nécessairement un domaine nodal de la fonction propre, il rencontre le bord. Donc $D_{i}$ est la réunion de son intérieur et d'un (ou plusieurs) intervalle ouvert du bord de $\Sigma$, donc $\chi\left(D_{i}\right) \leq 0$. Par conséquent, $\chi(\Gamma) \geq \chi(\Sigma)$.

Remarque 5.7. L'argument d'incompressibilité de l'ensemble nodal permet en fait de montrer un résultat plus fort mais dont nous ne ferons pas usage : l'ensemble nodal ne contient pas de courbe fermée qui borde un domaine.

5B. Majoration de multiplicité. Le but de cette section est de démontrer les bornes sur la multiplicité données par les théorèmes 1.11 et 1.15 .

Démonstration du théorème 1.11. La démonstration de l'inégalité (1.12) reprend la technique de N. Nadirashvili [1987] avec les améliorations que permet le problème de Steklov. On fixe un entier $k \geq 1$ et on note $m$ la multiplicité de la $k$-ième valeur propre. On se donne aussi un point $p \in \partial \Sigma$. Selon le lemme 5.2, on peut trouver une fonction non nulle dans le $k$-ième espace propre qui s'annule à l'ordre $m-1$ en $p$. Le point $p$ est donc l'extrémité de $m-1$ lignes nodales (théorème 5.1).

Parmi les lignes nodales d'extrémité $p$, il y en a au plus $1-\chi(\Sigma)$ dont les deux extrémités sont $p$. En effet, si on note $q$ le nombre de lignes allant de $p$ à $p$, la composante connexe de $p$ dans le graphe nodal aura une caractéristique d'Euler au plus égal à $1-q$, et donc $\chi(\Sigma) \leq 1-q$ d'après le théorème 5.5 . On déduit de cette remarque que $p$ est l'extrémité d'au moins $m+\chi(\Sigma)-2$ lignes distinctes.

On peut alors majorer $m$ en appliquant la formule d'Euler-Poincaré à la surface $\Sigma$. Contrairement à ce qui est fait dans la démonstration du théorème 5.5 , on considérera 
la surface ouverte, c'est-à-dire en ignorant le bord et les sommets du graphe nodal qui s'y trouve. On notera $\dot{\Gamma}$ le graphe ainsi obtenu. On a alors

$$
\chi(\Sigma)=\chi(\dot{\Gamma})+\sum_{i} \chi\left(D_{i}\right)
$$

Chaque domaine nodal vérifie $\chi\left(D_{i}\right) \leq 1$ donc $\sum_{i} \chi\left(D_{i}\right) \leq k+1$ selon le théorème de Courant. Comme $\dot{\Gamma}$ est la réunion d'au moins $m+\chi(\Sigma)-2$ lignes nodales homéomorphes à des intervalles ouverts, sa caractéristique d'Euler vérifie $\chi(\dot{\Gamma}) \leq$ $-m-\chi(\Sigma)+2$. On obtient finalement que

$$
m \leq k+3-2 \chi(\Sigma) \text {. }
$$

Avant d'entamer la démonstration du théorème 1.15, on va montrer une propriété des fonctions propres qui sera utile pour établir l'inégalité (1.17) :

Lemme 5.10. Soit $f$ une fonction propre de Steklov et $c>0$ un réel positif. Chaque composante connexe de l'ensemble $f^{-1}(]-c,+\infty[)$ contient au moins un domaine nodal positif de $f$. En particulier, si f est associée à la première valeur propre non nulle, cet ensemble est connexe.

Démonstration. Soit $D$ une composante connexe de $f^{-1}(]-c,+\infty[)$ qui ne contient pas de domaine nodal positif. Comme $f$ est harmonique, le maximum de $f$ sur $D$ est atteint en un point $x$ situé sur le bord de la surface.

On a nécessairement $f(x)<0$. En effet, si $f(x)>0$ alors $D$ contient un domaine nodal positif. Si $f(x)=0$, alors $x$ est l'extrémité d'une ligne nodale de $f$ qui traverse le domaine, donc $f$ change de signe dans $D$, en particulier elle prend des valeurs strictement positive dans $D$ ce qui est impossible.

Au point $x$, la fonction $f$ vérifie l'équation aux valeurs propres $\partial f / \partial v=\rho \sigma f$. Par conséquent, $(\partial f / \partial v)(x)$ est strictement négatif. Comme $v$ est un vecteur sortant normal au bord, on a une contradiction avec le fait que $x$ soit le maximum de $f$ sur $D$.

Pour finir, d'après ce qui précède le nombre de composantes connexes de $f^{-1}(]-c,+\infty$ [) est majoré par le nombre de domaines nodaux positifs de $f$. Or, si $f$ est associée à la première valeur propre non nulle elle a exactement deux domaines nodaux, un positif et un négatif. Par conséquent, $f^{-1}(]-c,+\infty[)$ n'a qu'une composante connexe.

Le théorème 1.15 repose sur un théorème de Sévennec :

Théorème 5.11 [Sévennec 2002, théorème 5]. Si $\Sigma$ est une surface close de caractéristique d'Euler strictement négative et $E$ un espace de fonction continues sur $\Sigma$ tel que pour toute fonction $f \in E \backslash\{0\}$, les ensembles $f^{-1}(] 0,+\infty[)$ et $f^{-1}([0,+\infty[)$ sont connexes et non vides, alors $\operatorname{Dim}(E) \leq 5-\chi(\Sigma)$. 
Remarque 5.12. Le principe du maximum assure que pour une fonction propre de Steklov $f \neq 0$, l'ensemble $f^{-1}([0,+\infty[)$ n'a pas de composante connexe disjointe de $f^{-1}(] 0,+\infty[)$.

Remarque 5.13. Pour appliquer le théorème 5.11 aux fonctions propres de $\sigma_{1}$, on utilise le théorème de Courant qui assure que ces fonctions propres ont exactement deux domaines nodaux. Or, G. Alessandrini [1998] a montré qu'il reste valable en dimension 2 si $g$ et $\gamma$ sont seulement $L^{\infty}$. Comme on n'a pas besoin de la régularité des fonctions propres sur le bord de la surface, on peut aussi supposer que $\rho$ est $L^{\infty}$.

Démonstration du théorème 1.15. On commence par l'inégalité (1.16). En collant un disque sur chaque composante du bord de $\Sigma$, on obtient une surface close $\bar{\Sigma}$ de caractéristique d'Euler $\bar{\chi}=\chi+l$.

On prolonge chaque fonction propre $f$ de la première valeur propre sur chacun de ces disques de la manière suivante : en notant $p$ le centre du disque et $x$ un point générique sur le bord, on fixe $f(p)=0$ et on interpole linéairement $f$ sur le segment $[p, x]$. La fonction $f$ ainsi prolongée est continue, et elle a deux domaines nodaux, un positif et un négatif (en effet, comme elle est de signe constant sur ] $p, x]$, les points intérieurs aux disques appartiennent aux mêmes domaines nodaux que les points de $\Sigma$ ). Compte tenu de la remarque 5.12, cette construction assure que les ensembles $f^{-1}\left(\left[0,+\infty[)\right.\right.$ et $\left.\left.f^{-1}(]-\infty, 0\right]\right)$ sont eux aussi connexes. D'après le théorème de Sévennec, cet espace est de dimension au plus $5-\bar{\chi}$. On a donc $m_{1} \leq 5-\bar{\chi}=5-\chi-l$.

Pour montrer l'inégalité (1.17), on va encore appliquer le théorème 5 de Sévennec mais à un espace plus grand : on considère l'espace $E$ engendré par les fonctions propres prolongées à $\bar{\Sigma}$ comme précédemment et par la fonction $\varphi$ définie par $\varphi \equiv 1$ sur $\Sigma, \varphi(p)=-1$ et en interpolant la fonction de manière affine sur les rayons du disque (comme on suppose que $l=1$, il n'y a qu'un disque et le point $p$ est unique). L'espace $E$ est constitué de fonction continues, on doit donc montrer que ces fonctions (à l'exception de la fonction nulle) ont exactement deux domaines nodaux, un positif et un négatif.

Une fonction de $E$ est une combinaison linéaire $f_{a, b}=a \cdot f+b \cdot \varphi, a, b \in \mathbb{R}$, $f$ étant une fonction propre prolongée à $\bar{\Sigma}$. Si $a$ ou $b$ est nul, il est clair que la fonction a un seul domaine nodal positif.

Sans nuire à la généralité, on peut supposer que $a=1$ et que $b$ est strictement positif. La fonction $f_{a, b}$ est égale à $f+b$ sur $\Sigma$ et $f_{a, b}(p)=-b$. Selon le lemme 5.10 appliqué à la fonction $f$, l'ensemble $f_{a, b}^{-1}(] 0,+\infty[)=f^{-1}(]-b,+\infty[)$ restreint à $\Sigma$ est connexe. Comme $f_{a, b}$ est affine sur les rayons du disque et négative en son centre, les points du disque où $f_{a, b}$ est positive sont connectés au domaine nodal positif sur $\Sigma$. La fonction $f_{a, b}$ n'a donc globalement qu'un seul domaine nodal positif. 
En restriction à $\Sigma$, la fonction $f_{a, b}$ peut avoir plusieurs domaines nodaux négatifs. Cependant, ils sont connectés à $p$ par des rayons sur lesquels $f_{a, b}$ est négative. Il n'y a donc qu'un seul domaine nodal négatif sur $\bar{\Sigma}$. Comme $E$ est de dimension $m_{1}+1$, l'application du théorème de Sévennec donne $m_{1}+1 \leq 5-\bar{\chi}=4-\chi$, soit $m_{1} \leq 3-\chi$.

5C. Première valeur propre en petit genre. On commencera dans ce paragraphe par démontrer le théorème 1.19 , puis on montrera séparément les calculs des trois valeurs de $m_{k}$ annoncées dans par le théorème 1.20, les deux premières dans ce paragraphe et la 3-ième dans le suivant.

Démonstration du théorème 1.19. Pour majorer la multiplicité il s'agit, comme dans [Fraser et Schoen 2015; Jammes 2014; Karpukhin et al. 2014], de se ramener à la démonstration utilisée dans le cas des opérateurs de Schrödinger sur les surfaces closes. La minoration découle du théorème 1.7.

Dans le cas de $\mathbb{T}_{p}^{2}$, on fixe un point $x$ intérieur à la surface et on suppose que la multiplicité de $\sigma_{1}$ est au moins 7. Il existe alors un espace de dimension 2 de fonctions propres qui s'annulent à l'ordre 3 en $x$, et on note $f_{\theta}$ le cercle unité de cet espace. Si on ferme la surface en contractant chaque bord de $\mathbb{T}_{p}^{2}$ en un point, les lignes nodales de $f_{\theta}$ qui atteignent le bord se prolongent sur le quotient en des courbes $C^{1}$ par morceaux. On peut alors reprendre les arguments de [Besson 1980] pour montrer que sur le quotient, l'ensemble nodal est formé d'exactement trois lacets disjoints non homotopes et aboutir à une contradiction en faisant varier $\theta$.

Sur $\mathbb{K}_{p}^{2}$, on se ramène de la même manière à la démonstration du théorème 4.1 de [Colin de Verdière 1987] en quotientant chaque bord sur un point.

Avant de traiter le cas de $\mathbb{P}_{2}^{2}$, on va montrer que $m_{1}\left(\mathbb{M}^{2}\right)=4$ par une méthode légèrement différente de celle du théorème 1.11. Cette démonstration servira de base pour les deux propositions qui vont suivre.

On commence par montrer qu'il existe une fonction propre pour laquelle il existe au moins 6 extrémités de lignes nodales qui rejoignent le bord. Soit $x$ un point du bord de $\mathbb{M}^{2}$. Si la multiplicité de $\sigma_{1}$ est supérieure ou égale à 5 , il existe une fonction propre pour laquelle $x$ est l'extrémité d'au moins quatre lignes nodales. S'il est l'extrémité d'au moins 5 lignes, alors il y a au moins six extrémités de lignes qui rejoignent le bord puisque ce nombre est toujours pair. S'il est l'extrémité d'exactement quatre lignes alors le signe de la fonction propre est constant le long du bord au voisinage de $x$, par conséquent la fonction propre change de signe ailleurs sur le bord (sinon la fonction est positive ou nulle partout sur le bord, donc partout sur $\mathbb{M}^{2}$ ) et il y a donc au moins six extrémités de lignes le long du bord. Si on définit une application $\mathbb{M}^{2} \mapsto \mathbb{P}^{2}$ par contraction du bord de $\mathbb{M}^{2}$ sur un point (qu'on notera encore $x$ ), on peut considérer l'image de la décomposition nodale de $\mathbb{M}^{2}$ par cette application : elle décompose $\mathbb{P}^{2}$ en deux domaines, et $x$ est l'extrémité 


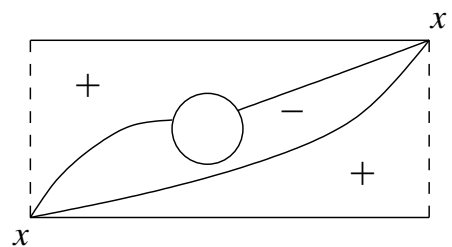

Figure 4. Décomposition nodale de $\mathbb{P}_{2}^{2}$.

de six arcs nodaux. Or Besson [1980] a montré qu'une telle décomposition de $\mathbb{P}^{2}$ est impossible.

\section{Proposition 5.14. $\quad m_{1}\left(\mathbb{P}_{2}^{2}\right)=4$.}

Démonstration. On représente $\mathbb{P}_{2}^{2}$ comme étant $\mathbb{M}^{2}$ privé d'un disque. On parlera de bord de $\mathbb{M}^{2}$ et de bord du disque pour désigner les deux bords de $\mathbb{P}_{2}^{2}$.

Le point de départ de la démonstration est le même que celle qui précède. On suppose que la multiplicité de $\sigma_{1}\left(\mathbb{P}_{2}^{2}\right)$ est 5 . Étant donné un point $x$ du bord de $\mathbb{M}^{2}$, il existe une fonction propre $f$ telle que $x$ soit localement l'extrémité de quatre lignes nodales. On a vu précédemment qu'il ne peut pas y avoir d'autres extrémités de lignes nodales sur le bord de $\mathbb{M}^{2}$. Le signe de $f$ le long de ce bord est donc constant et on le supposera positif. Comme les deux domaines nodaux de $f$ rencontrent le bord de $\mathbb{P}_{2}^{2}$, le domaine négatif rencontre nécessairement le bord du disque.

Si on considère un arc qui part orthogonalement de $x$ (avec par conséquent deux lignes nodales de part et d'autre, selon le théorème 5.1) et qui rejoint un autre point du bord en restant dans le domaine nodal positif, il découpe nécessairement le ruban $\mathbb{M}^{2}$ en un rectangle comme sur la figure 4 . La décomposition nodale de $\mathbb{P}_{2}^{2}$ est donc nécessairement topologiquement équivalente à celle de cette figure, à ceci près que le bord du disque peut être entièrement contenu dans le domaine nodal négatif.

Après avoir contracté le bord du disque sur un point qu'on notera $p$, on pourra donc toujours trouver dans $\mathbb{M}^{2}$ un lacet $\gamma_{x}$ d'extrémité $x$, unique à homotopie près, contenu dans le domaine nodal négatif, passant par $p$ et homotope à un générateur du groupe fondamental de $\mathbb{M}^{2}$.

Supposons maintenant qu'on déplace continûment le point $x$. Le lacet $\gamma_{x}$ peut alors se déformer continûment avec la contrainte de toujours passer par $p$. Si on fait faire à $x$ un tour complet du bord de $\mathbb{M}^{2}$ en partant d'un point $x_{0}$, on revient à la même décomposition de $\mathbb{P}_{2}^{2}$, donc le lacet $\gamma_{x}$ devient homotope au lacet initial $\gamma_{x_{0}}$. Or, chacun des arcs de $\gamma_{x_{0}}$ allant de $x_{0}$ à $p$ serait homotope à sa concaténation avec un générateur du groupe fondamental du bord, ce qui est impossible.

\section{Proposition 5.15.}

$$
m_{1}\left(\mathbb{T}_{1}^{2}\right)=5 \text {. }
$$

Démonstration. On procède par l'absurde en supposant que $\sigma_{1}\left(\mathbb{T}_{1}^{2}\right)$ est de multiplicité 6 pour une métrique $g$ et des densités $\rho, \gamma$ données. 
Soit $x$ un point du bord de $\mathbb{T}_{1}^{2}$. Selon le théorème 5.1 et le lemme 5.2, il existe une fonction propre $f$ qui s'annule à l'ordre 5 en $x$ et telle que cinq arcs nodaux partent de $x$. Notons que cette fonction est unique : dans le cas contraire, on pourrait en choisir une s'annulant à l'ordre 6 et on aurait une contradiction comme dans le théorème 1.11. Comme les extrémités de lignes nodales rejoignant le bord sont nécessairement en nombre pair, il existe une 6-ième extrémité en un point $x^{\prime}$ du bord, $x^{\prime}$ étant distinct de $x$ (sinon $f$ s'annulerait à l'ordre 6 en $x$ ).

Comme dans la démonstration précédente, on va déplacer le point $x$ le long du bord. Les arguments développés par Besson [1980] permettront d'aboutir à une contradiction. Si on contracte le bord sur un point - notons-le $\bar{x}$ - il y a six arcs nodaux partant de $\bar{x}$. On sait alors que l'ensemble nodal est la réunion de trois lacets non homotopes entre eux et qui ne s'intersectent qu'en $\bar{x}$ (cf. la démonstration du théorème 3.C.1 dans [Besson 1980]). Cette remarque permet alors de transposer le reste de la démonstration de [Besson 1980] : si $x$ se déplace continûment en partant d'un point $x_{0}$, on peut construire une homotopie entre les lignes nodales de la fonction propre $f_{x}$ correspondante et les lignes nodales de $f_{x_{0}}$ (il est crucial ici que $x \neq x^{\prime}$ quel que soit $x$ ). Après un tour complet de $x$ le long du bord, les classes d'homotopies des lignes nodales sont donc les conjuguées des lignes de $f_{x_{0}}$ par la classe d'homotopie du bord. Or elles doivent être identiques aux lignes nodales de $f_{x_{0}}$. Il y a contradiction car les classes d'homotopies des lignes nodales sont des classes d'homotopies non triviales dans le tore $\mathbb{T}^{2}$ obtenu par contraction du bord, elles ne commutent donc pas avec la classe du bord.

5D. Deuxième valeur propre du disque. Pour finir, on montre la majoration de $m_{2}\left(\mathbb{D}^{2}\right)$ annoncée par le théorème 1.21. On sait déjà, d'après le théorème 1.11 , que $m_{2}\left(\mathbb{D}^{2}\right) \leq 3$. On va supposer qu'il y a égalité pour aboutir à une contradiction.

On se donne une métrique $g$ et une densité $\gamma$ telle que la multiplicité de $\sigma_{2}\left(\mathbb{D}^{2}\right)$ soit 3. Selon le théorème de Courant, une fonction propre de $\sigma_{2}\left(\mathbb{D}^{2}\right)$ a deux ou trois domaines nodaux. Comme ces domaines sont simplement connexes, la décomposition nodale du disque est nécessairement topologiquement équivalente à l'une des trois indiquées sur la figure 5 .

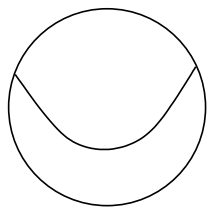

(a)

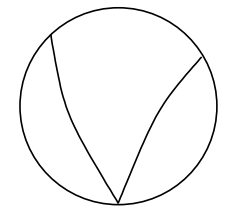

(b)

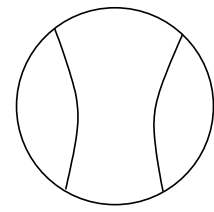

(c)

Figure 5. Domaines nodaux sur le disque. 
On considère la sphère unité de l'espace propre de $\sigma_{2}\left(\mathbb{D}^{2}\right)$, qu'on notera $S^{2}$, et on note respectivement $D_{a}$ (resp. $D_{b}$ et $D_{c}$ ) l'ensemble des fonctions propres de $S^{2}$ dont la décomposition nodale est celle de la figure 5(a) (resp. 5(b) et 5(c)). La contradiction découlera de l'étude de la partition de $S^{2}$ ainsi formée.

Commençons par noter que, comme la multiplicité est égale à 3 , le théorème 5.1 et le lemme 5.2 montrent qu'il existe nécessairement des fonctions propres réalisant la situation (b) de la figure 5 et donc $D_{b}$ est non vide. Plus précisément, pour chaque point $x$ de $\partial \mathbb{D}^{2}$, il existe une droite de fonction propre s'annulant à l'ordre 2 en $x$, et cette droite varie continûment avec $x$ (elle est unique car dans le cas contraire, on pourrait trouver une fonction s'annulant à l'ordre 3 en $x$ ). Comme cette droite coupe $S^{2}$ en deux points, l'ensemble $D_{b}$ est la réunion de deux cercles, l'un correspondant aux fonctions ayant un seul domaine nodal positif, l'autre à celles en ayant deux (en particulier, ces cercles sont disjoints).

Le complémentaire de $D_{b}$ est donc formé de trois composantes connexes, deux qui sont antipodales et homéomorphes à des disques et une homéomorphe à un cylindre. Comme $D_{c}$ a nécessairement deux composantes connexes antipodales, l'une formée des fonctions ayant un domaine nodal positif et l'autre des fonctions en ayant deux, $D_{c}$ est la réunion des deux disques et $D_{a}$ est le cylindre.

On choisit l'une des deux composantes de $D_{c}$, par exemple celle dont les fonctions ont deux domaines nodaux positifs, qu'on notera $D_{c}^{+}$, et on construit une application de $D_{c}^{+}$dans le cercle de la manière suivante : on se donne une orientation sur $\partial \mathbb{D}^{2}$, et pour chaque fonction de $D_{c}^{+}$on considère la paire de points de $\partial \mathbb{D}^{2}$ où la fonction s'annule en décroissant (par rapport à l'orientation du bord). Ces deux points sont nécessairement disjoints (sinon il y aurait au moins trois extrémités de lignes nodales se rejoignant en un même points du bord, ce qu'on a déjà exclu). On obtient ainsi une application continue partant de $D_{c}^{+}$et dont l'image est $S^{1} \times S^{1}$ privé de la diagonale et quotientée par $(x, y) \sim(y, x)$. Cette image est homotope à un cercle, ce qui permet de définir une application $D_{c}^{+} \rightarrow S^{1}$. Or, le long du bord de $D_{c}^{+}$, c'est-à-dire d'une des composantes de $D_{b}$, cette application est homotopiquement non triviale, ce qui fournit la contradiction cherchée.

\section{Remerciements}

Je remercie Y. Colin de Verdière et I. Polterovich pour leurs commentaires sur la première version de cet article, ainsi qu'un rapporteur anonyme dont les remarques ont permis d'améliorer le texte.

\section{Bibliographie}

[Alessandrini 1998] G. Alessandrini, "On Courant's nodal domain theorem", Forum Math. 10:5 (1998), 521-532. MR 99g:35091 Zbl 0909.35098 
[Appel et Haken 1976] K. Appel et W. Haken, "Every planar map is four colorable", Bull. Amer. Math. Soc. 82:5 (1976), 711-712. MR 54 \#12561 Zbl 0331.05106

[Bacher et Colin de Verdière 1995] R. Bacher et Y. Colin de Verdière, "Multiplicités des valeurs propres et transformations étoile-triangle des graphes", Bull. Soc. Math. France 123:4 (1995), 517-533. MR 96k:05129 Zbl 0845.05068

[Bandle 1980] C. Bandle, Isoperimetric inequalities and applications, Monographs and Studies in Mathematics 7, Pitman, Boston, 1980. MR 81e:35095 Zbl 0436.35063

[Besson 1980] G. Besson, "Sur la multiplicité de la première valeur propre des surfaces Riemanniennes”, Ann. Inst. Fourier (Grenoble) 30:1 (1980), 109-128. MR 81h:58059 Zbl 0417.30033

[Bollobás 1998] B. Bollobás, Modern graph theory, Graduate Texts in Mathematics 184, Springer, New York, 1998. MR 99h:05001 Zbl 0902.05016

[Bose 1939] R. C. Bose, "On the construction of balanced incomplete block designs", Ann. Eugenics 9 (1939), 353-399. MR 1,199b Zbl 0023.00102

[Cheng 1976] S. Y. Cheng, "Eigenfunctions and nodal sets", Comment. Math. Helv. 51:1 (1976), 43-55. MR 53 \#1661 Zbl 0334.35022

[Coxeter 1943] H. S. M. Coxeter, "The map-coloring of unorientable surfaces", Duke Math. J. 10 (1943), 293-304. MR 5,48f Zbl 0060.41602

[Fraser et Schoen 2011] A. Fraser et R. Schoen, "The first Steklov eigenvalue, conformal geometry, and minimal surfaces”, Adv. Math. 226:5 (2011), 4011-4030. MR 2012f:58054 Zbl 1215.53052

[Fraser et Schoen 2015] A. Fraser et R. Schoen, "Sharp eigenvalue bounds and minimal surfaces in the ball”, Invent. Math. (online publication May 2015), 1-68.

[Heawood 1890] P. J. Heawood, "Map-colour theorem", Quart. J. Pure Appl. Math. 24:96 (1890), 332-338. JFM 22.0562.02

[Heffter 1891] L. Heffter, "Ueber das Problem der Nachbargebiete", Math. Ann. 38:4 (1891), 477-508. MR 1510685 JFM 23.0543.01

[Jammes 2009] P. Jammes, "Sur la multiplicité des valeurs propres d'une variété compacte", pp. 1-11 dans Actes du Séminaire de Théorie Spectrale et Géométrie (Grenoble, 2007-2008), Séminaire de Théorie Spectrale et Géométrie 26, Université Grenoble I, Saint-Martin-d'Hères, 2009. MR 2011d:58075 Zbl 1235.58022

[Jammes 2014] P. Jammes, "Prescription du spectre de Steklov dans une classe conforme", Anal. PDE 7:3 (2014), 529-549. MR 3227426 Zbl 1304.35452

[Kagno 1935] I. N. Kagno, "A note on the Heawood color formula", J. Math. Phys. 14 (1935), 228-231. JFM 61.1347.01

[Karpukhin et al. 2014] M. Karpukhin, G. Kokarev et I. Polterovich, "Multiplicity bounds for Steklov eigenvalues on Riemannian surfaces”, Ann. Inst. Fourier (Grenoble) 64:6 (2014), 24812502. MR 3331172 Zbl 1321.58027

[Lovász et Schrijver 1998] L. Lovász et A. Schrijver, "A Borsuk theorem for antipodal links and a spectral characterization of linklessly embeddable graphs", Proc. Amer. Math. Soc. 126:5 (1998), 1275-1285. MR 98j:05059 Zbl 0886.05055

[Nadirashvili 1987] N. S. Nadirashvili, “Кратные собственные значения оператора Лаплаca", Mat. Sb. (N.S.) 133(175):2 (1987), 223-237. Translated as "Multiple eigenvalues of the Laplace operator" in Math. USSR Sb. 61 :1 (1988), 225-238. MR 89a:58113 Zbl 0672.35049

[Ringel 1974] G. Ringel, Map color theorem, Grundlehren der Mathematischen Wissenschaften 209, Springer, New York, 1974. MR 50 \#1955 Zbl 0287.05102 
[Ringel et Youngs 1968] G. Ringel et J. W. T. Youngs, "Solution of the Heawood map-coloring problem”, Proc. Nat. Acad. Sci. USA 60 (1968), 438-445. MR 37 \#3959 Zbl 0155.51201

[Robertson et al. 1995] N. Robertson, P. Seymour et R. Thomas, "Sachs' linkless embedding conjecture”, J. Combin. Theory Ser. B 64:2 (1995), 185-227. MR 96m:05072 Zbl 0832.05032

[Sévennec 1994] B. Sévennec, "Multiplicité du spectre des surfaces: une approche topologique", pp. 29-36 dans Actes du Séminaire de Théorie Spectrale et Géométrie (Grenoble, 1993-1994), Séminaire de Théorie Spectrale et Géométrie 12, Université Grenoble I, Saint-Martin-d'Hères, 1994. MR 1714546 Zbl 0909.58059

[Sévennec 2002] B. Sévennec, "Multiplicity of the second Schrödinger eigenvalue on closed surfaces", Math. Ann. 324:1 (2002), 195-211. MR 2003h:58045 Zbl 1053.58014

[Sylvester et Uhlmann 1990] J. Sylvester et G. Uhlmann, "The Dirichlet to Neumann map and applications", pp. 101-139 dans Inverse problems in partial differential equations (Arcata, CA, 1989), édité par D. Colton et al., SIAM, Philadelphia, 1990. MR 91d:35063 Zbl 0713.35100

[Uhlmann 2009] G. Uhlmann, "Electrical impedance tomography and Calderón's problem", Inv. Prob. 25:12 (2009), Article ID \#123011. Zbl 1181.35339

[Colin de Verdière 1987] Y. Colin de Verdière, "Construction de Laplaciens dont une partie finie du spectre est donnée”, Ann. Sci. École Norm. Sup. (4) 20:4 (1987), 599-615. MR 90d:58156 Zbl 0636.58036

[Colin de Verdière 1988] Y. Colin de Verdière, "Sur une hypothèse de transversalité d'Arnol'd", Comment. Math. Helv. 63:2 (1988), 184-193. MR 90c:58183 Zbl 0672.58046

[Colin de Verdière 1990] Y. Colin de Verdière, "Sur un nouvel invariant des graphes et un critère de planarité”, J. Combin. Theory Ser. B 50:1 (1990), 11-21. MR 91m:05068 Zbl 0742.05061

[Colin de Verdière 1998] Y. Colin de Verdière, Spectres de graphes, Cours Spécialisés 4, Société Mathématique de France, Paris, 1998. MR 99k:05108 Zbl 0913.05071

Received November 18, 2014. Revised September 8, 2015.

PIERRE JAMMES

CNRS, LJAD, UMR 7351

Univ. NicE SOPHia ANTIPOLIS

Parc Valrose

06100 NICE

FRANCE

pjammes@unice.fr 


\title{
PACIFIC JOURNAL OF MATHEMATICS
}

\author{
msp.org/pjm
}

Founded in 1951 by E. F. Beckenbach (1906-1982) and F. Wolf (1904-1989)

\section{EDITORS}

Don Blasius (Managing Editor)

Department of Mathematics

University of California

Los Angeles, CA 90095-1555

blasius@math.ucla.edu

\author{
Paul Balmer \\ Department of Mathematics \\ University of California \\ Los Angeles, CA 90095-1555 \\ balmer@math.ucla.edu \\ Robert Finn \\ Department of Mathematics \\ Stanford University \\ Stanford, CA 94305-2125 \\ finn@math.stanford.edu \\ Sorin Popa \\ Department of Mathematics \\ University of California \\ Los Angeles, CA 90095-1555 \\ popa@math.ucla.edu
}

\author{
Vyjayanthi Chari \\ Department of Mathematics \\ University of California \\ Riverside, CA 92521-0135 \\ chari@math.ucr.edu \\ Kefeng Liu \\ Department of Mathematics \\ University of California \\ Los Angeles, CA 90095-1555 \\ liu@math.ucla.edu \\ Jie Qing \\ Department of Mathematics \\ University of California \\ Santa Cruz, CA 95064 \\ qing@ cats.ucsc.edu
}

\section{PRODUCTION}

Silvio Levy, Scientific Editor, production@msp.org

\section{SUPPORTING INSTITUTIONS}

ACADEMIA SINICA, TAIPEI

CALIFORNIA INST. OF TECHNOLOGY

INST. DE MATEMÁTICA PURA E APLICADA

KEIO UNIVERSITY

MATH. SCIENCES RESEARCH INSTITUTE

NEW MEXICO STATE UNIV.

OREGON STATE UNIV.

\author{
STANFORD UNIVERSITY \\ UNIV. OF BRITISH COLUMBIA \\ UNIV. OF CALIFORNIA, BERKELEY \\ UNIV. OF CALIFORNIA, DAVIS \\ UNIV. OF CALIFORNIA, LOS ANGELES \\ UNIV. OF CALIFORNIA, RIVERSIDE \\ UNIV. OF CALIFORNIA, SAN DIEGO \\ UNIV. OF CALIF., SANTA BARBARA
}

\author{
Daryl Cooper \\ Department of Mathematics \\ University of California \\ Santa Barbara, CA 93106-3080 \\ cooper@math.ucsb.edu \\ Jiang-Hua Lu \\ Department of Mathematics \\ The University of Hong Kong \\ Pokfulam Rd., Hong Kong \\ jhlu@maths.hku.hk \\ Paul Yang \\ Department of Mathematics \\ Princeton University \\ Princeton NJ 08544-1000 \\ yang@math.princeton.edu
}

These supporting institutions contribute to the cost of publication of this Journal, but they are not owners or publishers and have no responsibility for its contents or policies.

See inside back cover or msp.org/pjm for submission instructions.

The subscription price for 2016 is US $\$ 440 /$ year for the electronic version, and $\$ 600 /$ year for print and electronic.

Subscriptions, requests for back issues and changes of subscribers address should be sent to Pacific Journal of Mathematics, P.O. Box 4163, Berkeley, CA 94704-0163, U.S.A. The Pacific Journal of Mathematics is indexed by Mathematical Reviews, Zentralblatt MATH, PASCAL CNRS Index, Referativnyi Zhurnal, Current Mathematical Publications and Web of Knowledge (Science Citation Index).

The Pacific Journal of Mathematics (ISSN 0030-8730) at the University of California, c/o Department of Mathematics, 798 Evans Hall \#3840, Berkeley, CA 94720-3840, is published twelve times a year. Periodical rate postage paid at Berkeley, CA 94704, and additional mailing offices. POSTMASTER: send address changes to Pacific Journal of Mathematics, P.O. Box 4163, Berkeley, CA 94704-0163.

PJM peer review and production are managed by EditFLOW ${ }^{\circledR}$ from Mathematical Sciences Publishers.

\section{PUBLISHED BY}

\section{mathematical sciences publishers \\ nonprofit scientific publishing}

http://msp.org/

(C) 2016 Mathematical Sciences Publishers 


\section{PACIFIC JOURNAL OF MATHEMATICS}

Volume 282 No. $1 \quad$ May 2016

On the half-space theorem for minimal surfaces in Heisenberg space

Tristan Alex

Extending smooth cyclic group actions on the Poincaré homology sphere 9

NIMA ANVARI

A short proof of the existence of supercuspidal representations for all reductive $p$-adic groups

RAPHAËL BEUZART-PLESSIS

Quantum groups and generalized circular elements

MICHAEL BRANNAN and KAY KIRKPATRICK

Volumes of Montesinos links

KathleEn Finlinson and Jessica S. PurCell

Minimal surfaces with two ends which have the least total absolute curvature

SHOICHI FUJIMORI and TOSHIHIRO SHODA

Multiplicité du spectre de Steklov sur les surfaces et nombre chromatique

\section{PIERRE JAMMES}

$E$-polynomial of the $\mathrm{SL}(3, \mathbb{C})$-character variety of free groups

SEAN LAWTON and VicEnTE MuÑOZ

The Blum-Hanson property for $\mathscr{C}(K)$ spaces

PASCAL LEFÈVRE and ÉTIENNE MATHERON

Crossed product algebras and direct integral decomposition for Lie supergroups

KARL-HERMANN NEEB and HADI SALMASIAN Associated primes of local cohomology modules over regular rings

TONY J. PUTHENPURAKAL 